\title{
Radiofrequency Signal in the Radiofrequency Ablation System
}

\author{
Rui-rui WANG ${ }^{1}$, Qun NAN ${ }^{1 *}$, Zhen TIAN ${ }^{1}$, Xiao-hui NIE ${ }^{1}$ and \\ Tong DONG ${ }^{1}$ \\ ${ }^{1}$ College of Life Science and Bioengineering, Beijing University of Technology, Beijing, \\ 100124, China. \\ ${ }^{*}$ Corresponding author: Qun Nan, Email:nanqun@bjut.edu.cn
}

Keywords: Radiofrequency ablation system, Instrument, Signal, Temperature control.

\begin{abstract}
Radiofrequency ablation technique is one of the tumor hyperthermia methods and has been widely used in clinical treatment because of its small traumatic, better curative effect and others advantages. Recently, the common automatic control of radiofrequency ablation system is temperature control model that only used to control electrode tip temperature by controlling center of the output power to prevent cooking, rather than monitoring temperature of tumor edge, leading to the result is not accurate. So for the purpose of being ablated completely without damaging the normal tissue, this paper puts forward with double temperature controlling system to monitor the electrode tip and the tumor edge temperature at the same time, and control the output power according to the change of the temperature, in order to achieve the purpose of accurate ablation. Now The design of radiofrequency ablation signal have been finished, including the final output signal peak-to-peak voltage of $140 \mathrm{~V}$, current $0.3 \mathrm{~A}$, total power adjusted in $2 \mathrm{~W}$ to $18 \mathrm{~W}$.
\end{abstract}

\section{Introduction}

\section{Radiofrequency Ablation (RFA)}

Presently, a growing number of people are becoming to concern the treatment and prevention of cancer because of its increasing incidence year by year. Cancer treatment methods are becoming more and more diverse and effective, from surgery, radiotherapy and chemotherapy to cellular immune therapy, then to ablation in recent years. Ablation method also had a great development, such as thermal ablation, cold ablation and laser ablation etc. And radiofrequency thermal ablation method is one of widely used ablation methods.

Radiofrequency (RF) is a kind of electromagnetic waves or high frequency current, and its mainly effect is organism thermal in the biosome. After starting the RF power source, high frequency sine wave current flow into body tissue from active electrode and discharge from the dispersive electrode. The human body is composed of many complex structures of organic and inorganic materials, containing lots of the dielectric in the body fluids, such as ion, water, colloid particles, etc. Ion is fast vibrating back and forth between the two electrodes under the high frequency oscillation. Due to the size of the various ions, mass, charge and different moving rates, ions friction with each other and produce biological heat, leading to local fever [1]. Due to tumor poor heat dissipation, the tumor tissue temperature is higher than the adjacent normal tissue, and the cancer is sensitive to heat, high heat killing cells or producing coagulation zone, and relative keeping normal tissue well. The ideal coagulation zone should be spherical 
or flat spherical shape. Recently, this method is widely used in clinic for tumor treatment [2] .

\section{Radiofrequency Ablation System and Temperature Control Module}

Radiofrequency thermal ablation system is composed by radiofrequency generator (including power amplification module) measurement, the needle electrode (including skin electrode) and control section, including computer [3,4]. The computer is also known as the host computer while the remaining part is called lower position machine system and the lower machine system controlled by PC. Ablation electrode is the core component of radiofrequency ablation instrument, because it directly affects the size and shape of the coagulation necrosis. Insert the needle electrode into cancer zone, and lead out current with skin electrode, forming a current loop. Ablation electrode will directly piece into the diseased tissue under the guidance of ultrasound or CT. The change of the tumor tissue parameters such as temperature, impedance are monitored by measurement and control unit that automatically adjust the output power of radiofrequency ablation, makes the system can automatically control, forming a closed loop automatic control, so as to make the tumor tissue quickly produces a wide range of coagulation necrosis. But now there are lots of problems existed in ablation equipment temperature and power control, which directly affect the melting effect. So this paper is based on this target to design a more effective radiofrequency ablation temperature and power control system, measuring temperature ablation area center and edge at the same time [5].

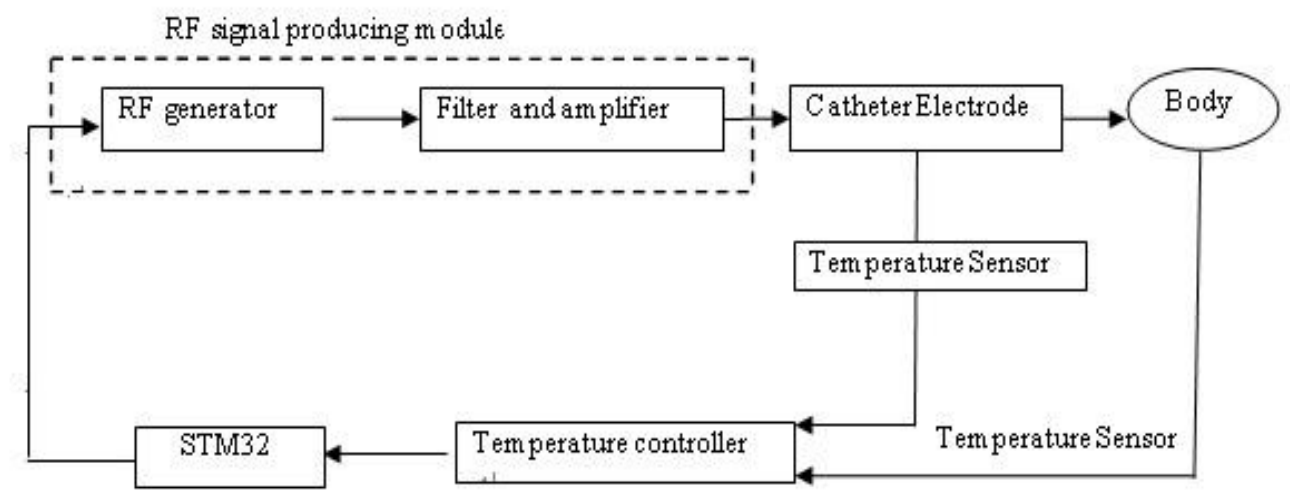

Figure 1. RFA system

Intelligent temperature control system is generally based on single-chip microcomputer control or DSP control. Single chip microcomputer is widely used in the automatic control system and the detection as its programmable.

In terms of software algorithm design of the control system, much of them are based on fuzzy control algorithm [6,7], neural network algorithm and genetic algorithm, and the PID algorithm design[8-11]. But we often encountered in the process of production of temperature control system with large lag. If we simple correct using the algorithm of PID temperature control system, there has a large high frequency disturbance, adjust the time, the PID parameter setting difficult, and the disadvantages such as larger overshoot amount. So as to complement each other's advantages, PID algorithm and other algorithms are used together. 


\section{Design and Construction of Instrument}

In our design of radiofrequency ablation system, we use STM32 MCU as a core component for the temperature control. System's main components are composed by following parts: RF signal producing module, J type and PT100 as temperature sensor, STM32F4 microcontroller (including display circuit), and temperature and power control circuit.

(1) The RF signal producing module: Design a power adjusting the RF signal occurs that can produce $460 \mathrm{KHz}$ sine wave.

(2) J type and PT100 as temperature sensor: Using J type and PT100 as temperature sensor, the temperature of central area and then send into the AD conversion circuit, after converted to digital signals sending into single chip microcomputer.

(3) STM32F4 microcontroller (including display circuit), as well as temperature and power control circuit.

In the temperature control system, STM32 microcontroller is the core part of the control system. Two temperature signals will be processed and shown in the display circuit after sending into MCU at the same time. Firstly, the two-way signal compared with their own set of threshold, then carry through the priority judgment, finally the judgment result will be sent into the controller to control the operation, which trigger the fuzzy PID program and executive power control unit according to the amount of control, so as to make temperature maintained at the required temperature.

\section{Radiofrequency Ablation Signal Producing Module}

Signal producing module schematic diagram is showing in Figure2:

Firstly, Sine wave signal is generated by AD9834, and then it will be send to the filter for filtering. If the sine wave is filtered thoroughly, it will be send into the power amplifier power to amplify, achieving need power. Finally the signal went into ablation electrode to ablate. In order to change the output power, we use AD5660 to change amplitude of the AD9834 output signal, to ultimately change the result of the output signal power. And AD5660 output control signal is controlled by single chip microcomputer input signals, achieving the corresponding changes. System clock provides clock signal for AD9834 .The power supply for single chip microcomputer, the system clock and AD chip stable electricity.

The sine wave signal generated is $460 \mathrm{KHz}$, and voltage of peak-to-peak value is up to $140 \mathrm{~V}$, current $0.3 \mathrm{~A}$ after power amplifier amplification. An average power is of around $15 \mathrm{~W}$.

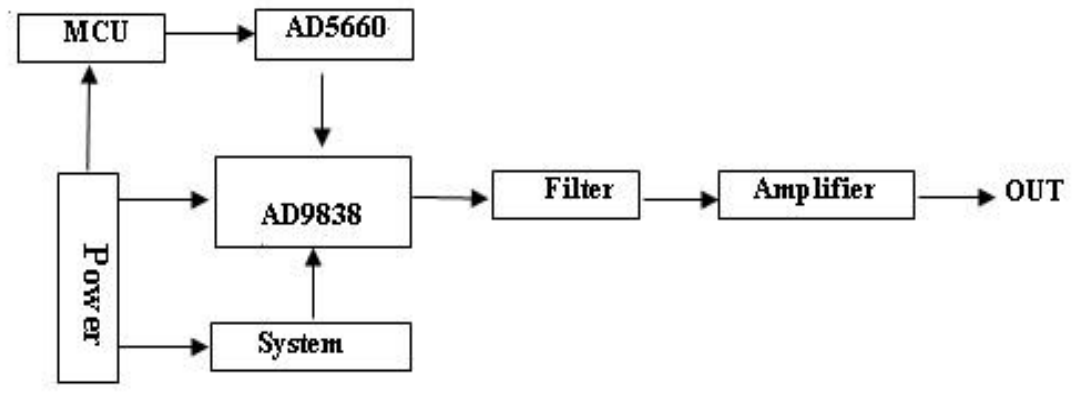

Figure2. RFA signal producing module 
The following (Figure3) is the physical and generate signal drawings

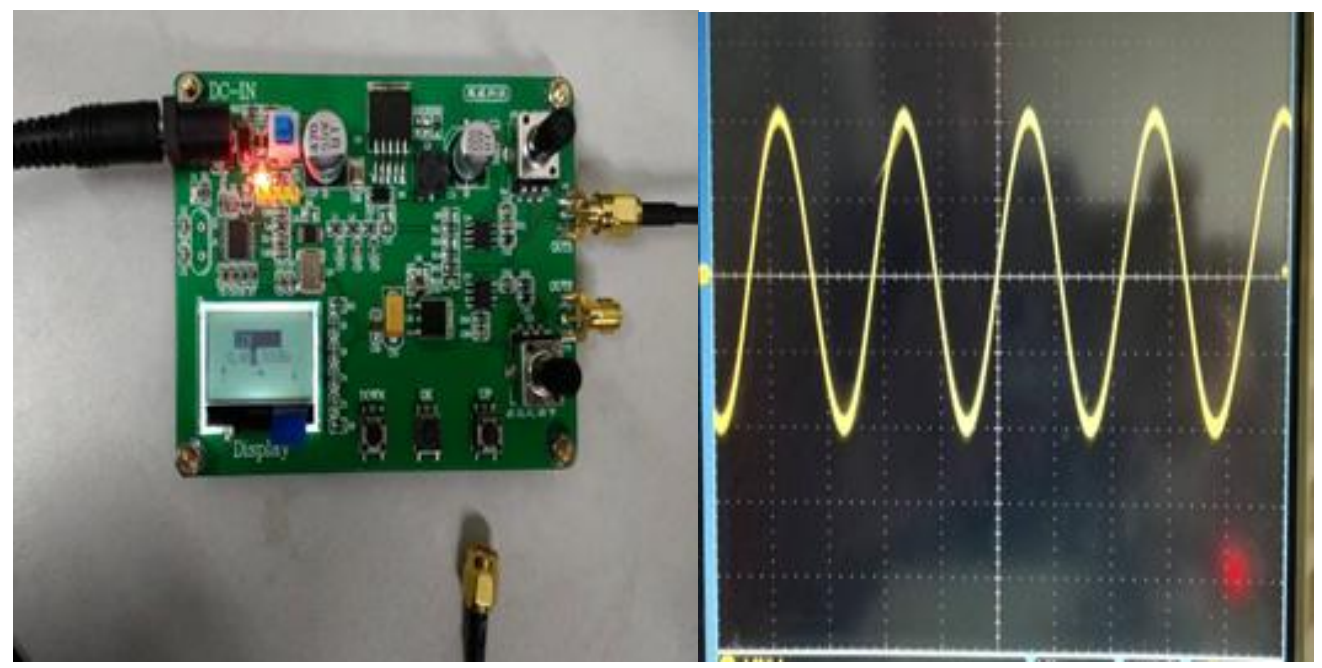

Figure3. RFA signal producing module and sine wave

\section{Conclusion}

The goal of this work was to create a new a set of radiofrequency ablation system, including the generation of RF signal, filter and amplification and temperature real-time monitoring and control system, etc. Now, we have finished generation, filtering and amplification of the RF signal, making the final biggest output signal voltage of $140 \mathrm{~V}$, current $0.3 \mathrm{~A}$, total power adjusted in $2 \mathrm{~W}$ to $18 \mathrm{~W}$ (as shown in figure $3)$. We have reached the preliminary setting for radiofrequency ablation instrument, and the further research is underway.

\section{Acknowledgement}

This research is supported by Beijing Natural Science Foundation (3162006), National Science Foundation of China (31070754), Beijing Municipal Commission of Education Project Scientific and Technological Program (KM201410005028), the Importation and Development of High-Caliber Talents Project of Beijing Municipal Institutions.

\section{References}

[1] J .Grepl, M.Penhaker ,M.Hlavackova, et al. Layout optimization of electrodes for radiofrequency ablation instrument[C].Applied Machine Intelligence and Informatics (SAMI), 2013 IEEE 11th International Symposium on. 2013:165-168.

[2] H.C. Patel, P.S.Dhillon, F. Mahfoud, et al. The biophysics of renal sympathetic denervation using radiofrequency energy [J]. Clinical Research in Cardiology Official Journal of the German Cardiac Society, 2014, 103(5):337-44.

[3] E.Lermite, J. Lebigot, F.Oberti et al. Radiofrequency thermal ablation of liver carcinoma: Prospective study of 82 lesions[J].Gastroentérologie clinique et biologique, 2006, 30(1): 130-135.

[4] D. Zheng, et al. Cool-tip Radiofrequency Ablation Therapy Instrument Based on Impedance Control Algorithm [J]. Journal of biomedical engineering.32.4 (2015): 905-909. 
[5] V. Lamonaca, A .Virga, M I. Minervini, et al. Cystic echinococcosis of the liver and lung treated by radiofrequency thermal ablation: An ex-vivo pilot experimental study in animal models [J]. Journal of Nervous \& Mental Disease, 1944, 100(1):37-38.

[6] A J. Vander Wal. Application of fuzzy logic control in industry [J]. Fuzzy Sets and Systems, 1995, 74(1): 33-41.

[7] K.Erkorkmaz, Y .Altintas. High speed CNC system design. Part I: jerk limited trajectory generation and quintic spline interpolation $[\mathrm{J}]$. International Journal of Machine Tools \& Manufacture, 2001, 41(9):1323-1345.

[8] Z. Runjing, X.Hongwei ,Zhijun. Design of fuzzy temperature control system based on fpga[C].Control, Automation and Systems Engineering (CASE), 2011 International Conference on. IEEE, 2011: 1-4.

[9] M .K.halid, S .Omatu. A neural network controller for a temperature control system [J]. Control Systems, IEEE, 1992, 12(3): 58-64.

[10] C.T. Lin, C S G. Lee. Neural-network-based fuzzy logic control and decision system [J]. Computers, IEEE Transactions on, 1991, 40(12): 1320-1336.

[11] C. J .Lin. A GA-based neural fuzzy system for temperature control [J]. Fuzzy Sets and Systems, 2004, 143(2): 311-333. 